\title{
Probabilistic Multi-Shape Representation Using an Isometric Log-Ratio Mapping
}

\author{
Neda Changizi and Ghassan Hamarneh \\ Medical Image Analysis Lab, Simon Fraser University, Canada \\ $\{$ nca19, hamarneh\}@cs.sfu.ca
}

\begin{abstract}
Several sources of uncertainties in shape boundaries in medical images have motivated the use of probabilistic labeling approaches. Although it is well-known that the sample space for the probabilistic representation of a pixel is the unit simplex, standard techniques of statistical shape analysis (e.g. principal component analysis) have been applied to probabilistic data as if they lie in the unconstrained real Euclidean space. Since these techniques are not constrained to the geometry of the simplex, the statistically feasible data produced end up representing invalid (out of the simplex) shapes. By making use of methods for dealing with what is known as compositional or closed data, we propose a new framework intrinsic to the unit simplex for statistical analysis of probabilistic multi-shape anatomy. In this framework, the isometric logratio (ILR) transformation is used to isometrically and bijectively map the simplex to the Euclidean real space, where data are analyzed in the same way as unconstrained data and then back-transformed to the simplex. We demonstrate favorable properties of ILR over existing mappings (e.g. LogOdds). Our results on synthetic and brain data exhibit a more accurate statistical analysis of probabilistic shapes.
\end{abstract}

\section{Introduction}

Numerous sources of uncertainties exist in shape boundaries including tissue heterogeneity [1], image acquisition artifacts, segmentation by multiple-raters, and image segmentation algorithms intentionally designed to output fuzzy results 223. It is important not to ignore these uncertainties in subsequent analyses and decision-making [14]. In order to capture information concerning uncertainty in addition to the shape of multiple structures, probabilistic multi-shape representations have been proposed. Being able to perform statistical analysis on these probabilistic multi-shape representations is important in understanding normal and pathological geometrical variability of anatomical structures.

Multi-shape non-probabilistic (crisp) representations have been proposed by Tsai et al. 5], and Babalola and Cootes [6]. Leventon et al. adopted the signed distance map (SDM) representation and performed linear principal component analysis (PCA) to extract shape statistics [7]. Besides the fact that SDMs are not designed to encode uncertainty, the main disadvantage of this method is that it is not obvious how to impose a vector space structure on SDMs. This

T. Jiang et al. (Eds.): MICCAI 2010, Part III, LNCS 6363, pp. $563-570,2010$.
(C) Springer-Verlag Berlin Heidelberg 2010 
is usually dealt with by projecting samples from the distribution given by the PCA coefficients back onto the manifold of valid SDMs [8]. Pohl et al. used the logarithm of the odds ratio (LogOdds) method to place probability atlases in a linear vector space 9]. In 9, linear PCA was done on layered SDMs (interpreted as LogOdds maps) to build a statistical shape atlas of brain structures. This means that, while the layered SDMs are exactly the logarithm-of-odds representations, results after algebraic manipulation in the logarithm-of-odds space often yield invalid SDMs (but still valid logarithm-of-odds representations). Using such results, computing probabilities as described in 9 may yield erroneous likelihoods, since vector operations are not closed under the set of SDMs. Malcolm et al. proposed a mapping of labels to vertices of a regular simplex which form the basis of a convex linear structure [10. Hamarneh and Changizi proposed a proper inverse function for label space representation based on barycentric coordinates [11. Performing linear PCA on the label space representation then exploring the variational modes may result in new invalid points out of the simplex (e.g. negative probabilities or sums exceeding unity). A possible remedy is to project such points onto the simplex 12. However, this causes points along modes of variation (from some point onward) to collapse onto a single point, which gives improper results and violates the indefinite Gaussian distribution assumption of PCA. Another way to circumvent the problem is to impose some limits when exploring the modes of variation to force the points to stay within the simplex. This is undesired, however, as it renders traditional statistical exploration invalid, e.g. one may no longer be able to explore the variability within \pm 3 standard deviations from the mean.

Compositional or closed data are multivariate data with positive values that sum up to a constant, usually chosen as 1 . Compositional data has arisen in many different disciplines such as geology (mineral compositions of rocks), environometrics (pollutant compositions), economics (household budget compositions), etc. [13. We extend this list of application areas to anatomical compositions within image pixels, resulting from the aforementioned uncertainties (e.g. partial volume effect) 1234 .

Standard statistical techniques may lead to misleading results if they are directly applied to closed data. Statistical analysis of compositional data has been a developing area since 1986, when Aitchison introduced two transformations of compositional data to real space: the additive log-ratio (ALR) and the centered log-ratio (CLR) transformations, as well as a proper distance metric in the simplex 13. Aitchison applied classical statistical analysis to the transformed observations, using ALR for modeling and CLR for those techniques based on a metric. The underlying reason was that ALR does not preserve distances, whereas CLR preserves distances but leads to a singular covariance matrix [14. Egozcue et al. defined a new isometric log-ratio (ILR) transformation [15], which is an isometry between the simplex and the real space of the same dimension, avoiding the drawbacks of both ALR and CLR.

In this paper, we propose to consider the algebraic-geometrical structure (Hilbert space) of the simplex, based on operations of perturbation, power 
transformation and the Aitchison inner product [13]. Through the ILR transformation, we develop techniques for statistical analysis of probabilistic multi-shape representations, which are not only intrinsic to the simplex but are bijective, isometric, and yielding non-singular covariance.

\section{Methods}

A sample space of compositional data, such as the probabilistic representation $\mathbf{x}=\left[x_{1}, x_{2}, \ldots, x_{n}\right]$ of a pixel with $n$ labels, is an $(n-1)$-dimensional unit simplex $S^{n-1} \subset R^{n}$ given as

$$
S^{n-1}=\left\{\mathbf{x}=\left[x_{1}, x_{2}, \ldots, x_{n}\right] \in \mathbb{R}^{n} \mid x_{i}>0, i=1,2, \ldots, n ; \sum_{i=1}^{n} x_{i}=1\right\} .
$$

Two basic operations were defined on the simplex by Aitchison [13. (i) Perturbation, which is analogous to displacement or translation in real space and is defined for any $\mathbf{x}, \mathbf{y} \in S^{n-1}$ as

$$
\mathbf{x} \oplus \mathbf{y}=\mathcal{C}\left[x_{1} y_{1}, x_{2} y_{2}, \ldots, x_{n} y_{n}\right]
$$

(ii) Power transformation, which is analogous to scalar multiplication in real space and is defined for a vector $\mathbf{x} \in S^{n-1}$ and a scalar $\alpha \in \mathbb{R}$ as

$$
\alpha \odot \mathbf{x}=\mathcal{C}\left[x_{1}^{\alpha}, x_{2}^{\alpha}, \ldots, x_{n}^{\alpha}\right] .
$$

In equations (2) and (3), $\mathcal{C}$ is the closure operation defined for a vector $\mathbf{z}=$ $\left[z_{1}, z_{2}, \ldots, z_{n}\right] \in \mathbb{R}_{+}^{n}$ as

$$
\mathcal{C}(\mathbf{z})=\left[\frac{z_{1}}{\sum_{i=1}^{n} z_{i}}, \frac{z_{2}}{\sum_{i=1}^{n} z_{i}}, \ldots, \frac{z_{n}}{\sum_{i=1}^{n} z_{i}}\right] .
$$

The internal simplicial operation of perturbation, and the external operation of powering define an $(n-1)$-dimensional vector space (indeed a Hilbert space) on $S^{n-1}$ [16 17 18 19]. The structure can be extended to produce a metric vector space by the introduction of the simplicial metric $d_{S}$ defined by Aitchison as [13]

$$
d_{S}(\mathbf{x}, \mathbf{y})=\left[\frac{1}{n} \sum_{i<j}\left(\ln \frac{x_{i}}{x_{j}}-\ln \frac{y_{i}}{y_{j}}\right)^{2}\right]^{\frac{1}{2}}=\left[\sum_{i=1}^{n}\left(\ln \frac{x_{i}}{g(\mathbf{x})}-\ln \frac{y_{i}}{g(\mathbf{y})}\right)^{2}\right]^{\frac{1}{2}},
$$

where $g(\mathbf{x})=\left(\prod_{i=1}^{n} x_{i}\right)^{\frac{1}{n}}$ is the component-wise geometric mean of the composition. An inner product $\langle\mathbf{x}, \mathbf{y}\rangle_{S}$ and a norm $\|\mathbf{x}\|_{S}^{2}=\langle\mathbf{x}, \mathbf{x}\rangle_{S}$, consistent with this metric, complete the Euclidean structure of the simplex:

$$
\langle\mathbf{x}, \mathbf{y}\rangle_{S}=\frac{1}{D} \sum_{i<j} \ln \frac{x_{i}}{x_{j}} \ln \frac{y_{i}}{y_{j}}=\sum_{i=1}^{D} \ln \frac{x_{i}}{g(\mathbf{x})} \ln \frac{y_{i}}{g(\mathbf{y})} .
$$



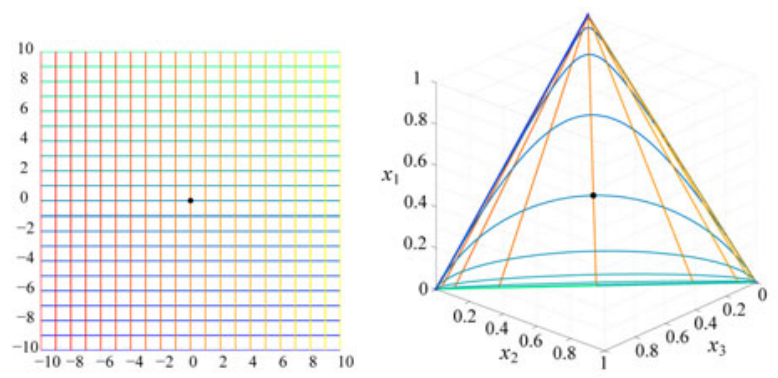

Fig. 1. Left: Orthogonal grids of lines in $\mathbb{R}^{2}$. Right: Orthogonal compositional lines (colors correspond) in $S^{2}$, equally spaced by 1 unit in Aitchison distance 13 .

In any finite dimensional Hilbert space, a geodesic curve connecting two points is understood to be the only continuous curve whose length is minimum with respect to the distance metric of the space. Such a geodesic is a segment of a 'straight' line within the geometry of the space considered. To avoid confusion, straight lines with respect to the Aitchison geometry are called compositional lines (Figure 1). A compositional line going from $\mathbf{x}_{0}$ to $\mathbf{x}(t)$, with the leading vector $\mathbf{p}$, in $S^{n-1}$ is given by

$$
\mathbf{x}(t)=\mathbf{x}_{0} \oplus(t \odot \mathbf{p}) \quad t \in \mathbb{R}, \mathbf{x}_{0}, \mathbf{p} \in S^{n-1} .
$$

Three log-ratio transformations have been proposed so far to map the simplex to the Euclidean real space. (i) The ALR transformation [13] (the same as the LogOdds method [9]):

$$
\operatorname{alr}: S^{n-1} \rightarrow \mathbb{R}^{n-1}, \quad \operatorname{alr}(\mathbf{x})=\left[\ln \frac{x_{1}}{x_{n}}, \ln \frac{x_{2}}{x_{n}}, \ldots, \ln \frac{x_{n-1}}{x_{n}}\right] .
$$

This transformation is asymmetric in the parts of the composition. By changing the part in the denominator, we obtain different alr transformations. Although all the statistical procedures are invariant under a permutation of the compositional parts [13. But the main drawback of ALR is the fact that it is not an isometric (but an isomorphic or bijective) transformation from the simplex, with the Aitchison metric, onto the real space, with the ordinary Euclidean metric. In fact, alr coefficients are coordinates in an oblique basis, something that affects distances if the usual Euclidean distance is computed from the alr coordinates. (ii) The CLR transformation [13]:

$$
\operatorname{clr}: S^{n-1} \rightarrow \mathbb{R}^{n}, \quad \operatorname{cl}(\mathbf{x})=\left[\ln \frac{x_{1}}{g(\mathbf{x})}, \ln \frac{x_{2}}{g(\mathbf{x})}, \ldots, \ln \frac{x_{n}}{g(\mathbf{x})}\right]
$$

CLR is symmetrical in the components, but since the sum of the components has to be zero, this transformation leads to a singular covariance matrix. CLR is an isometry but between the $(n-1)$-dimensional simplex $S^{n-1}$ and a subspace of real space $\mathbb{R}^{n}$. In this paper, we propose to adopt the third transformation: 
(iii) The ILR transformation [15]:

$$
i l r: S^{n-1} \rightarrow \mathbb{R}^{n-1}, \quad \mathbf{y}=i l r(\mathbf{x})=\left[\left\langle\mathbf{x}, \mathbf{e}_{1}\right\rangle_{S},\left\langle\mathbf{x}, \mathbf{e}_{2}\right\rangle_{S}, \ldots\left\langle\mathbf{x}, \mathbf{e}_{n-1}\right\rangle_{S}\right] .
$$

ILR is based on the choice of orthonormal bases $\mathbf{e}_{i}, i=\{1,2, \ldots, n-1\}$ of the simplex $S^{n-1}$ that can be calculated using the Gram-Schmidt procedure. The ILR transformation of any $\mathbf{x} \in S^{n-1}$ gives the coordinates of $\mathbf{x}$ with respect to the basis $\mathbf{e}_{1}, \mathbf{e}_{2}, \ldots, \mathbf{e}_{n-1}$. The inverse ILR transformation corresponds to the expression of $\mathbf{x}$ in the reference basis of $S^{n-1}$ :

$$
\mathbf{x}=i l r^{-1}(\mathbf{y})=\bigoplus_{i=1}^{n-1}\left(\mathbf{y}_{i} \odot \mathbf{e}_{i}\right), \quad \text { where } \quad y_{i}=\left\langle\mathbf{x}, \mathbf{e}_{i}\right\rangle_{S} .
$$

\section{Results}

We first show the shortcomings of ALR and CLR in comparison to ILR. We created 10,000 random pairs $(\mathbf{x}, \mathbf{y})$ of probability vectors for different number of labels $n=2,3,4$, sampled from a Dirichlet distribution with parameters $\alpha_{1}=\cdots=\alpha_{n}=1$, giving a uniform distribution within the $(n-1)$-dimensional open simplex and zero elsewhere [20]. We calculated the distance between the two vectors in each pair in different spaces: (a) In $S^{n-1}$, using the simplicial metric $d_{S}(\mathbf{x}, \mathbf{y})$ from equation (5), (b) Euclidean distance between the ILR-transformed vectors, i.e. $|i \operatorname{lr}(\mathbf{x})-i \operatorname{lr}(\mathbf{y})|_{2}$, (c) $|\operatorname{alr}(\mathbf{x})-\operatorname{alr}(\mathbf{y})|_{2}$, and (d) $|\mathbf{x}-\mathbf{y}|_{2}$. The scatter plots in Figure 2 show the relation between the different distances. Note how only the distances of the proposed ILR-transformed vectors remain faithful to the proper simplicial distance (diagonal line in the leftmost plot). We also show this difference in distance calculations by starting with a set of probability vectors forming a unit circle, according to the simplex geometry, in $S^{2}$ and noting the isometry (between $S^{2}$ and $\mathbb{R}^{2}$ ) only when transforming these vectors to ILR space, but not ALR or CLR (Figure 3).
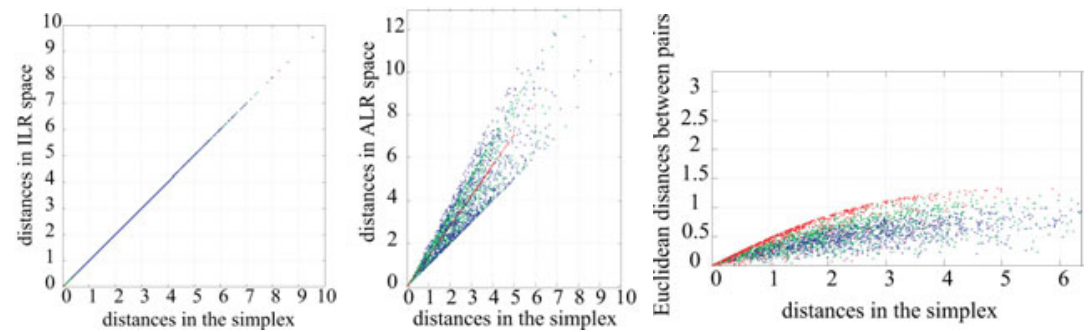

Fig. 2. Comparing distances between 10,000 random pair of probability vectors calculated in the ILR space (Left), ALR space (Center), and Euclidean distances between probability vectors (Right) vs. using the proper simplicial metric. Red, green, and blue points correspond to distances between vectors with 2,3 , and 4 labels, respectively. For ILR, all colored points collapse onto the diagonal. 

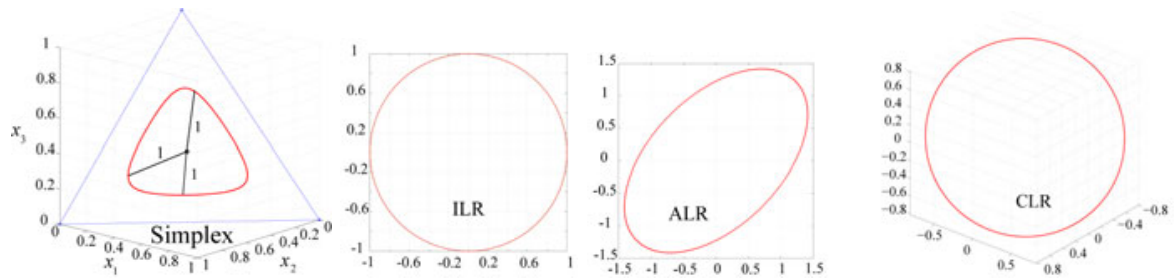

Fig. 3. Left to right: Unit circle according to the simplex $\left(S^{2}\right)$ geometry, transformed isometrically to a unit circle in ILR space, distances are unpreserved in ALR space whereas an extra dimension is added in CLR space

Next, we show that performing statistical analysis (e.g. PCA) in ILR and ALR spaces lead to different results. In the first experiment, we sampled a simple curve in $S^{2}$ and transformed the samples to ILR and ALR. After performing PCA in the Euclidean spaces of ILR and ALR, we back-transformed the results to the simplex. We also performed PCA on the label space representation of the samples. It is shown that moving along the modes of variation obtained from ALR and ILR transformations result in different probabilities. It is also obvious that, in label space, it is not allowable to move freely along the modes since, at some point, the mode will leave the simplex (e.g. negative probabilities). This experiment is repeated for a random set of points in $S^{2}$ (Figure 4).
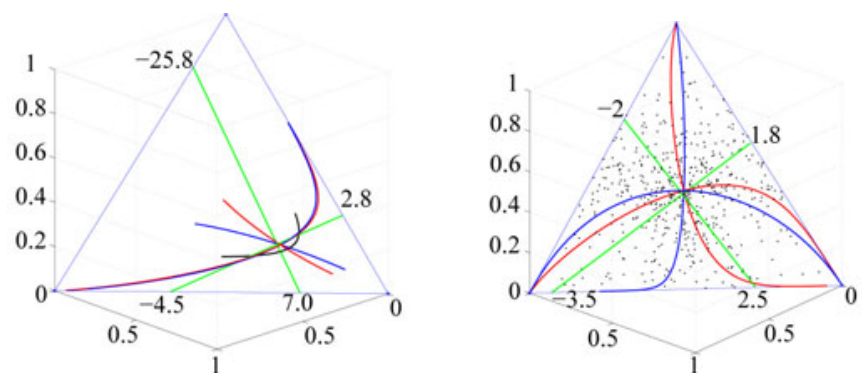

Fig. 4. For a given set of points (black) in $S^{2}$, new points along the modes of variation $(-10$ to $10 \sqrt{\lambda}$ ) are obtained using ILR (red) and ALR (blue), where $\lambda$ is the variance explained by that mode. Note that when the label space method is used (green), limits are imposed (factors of $\sqrt{\lambda}$ for each mode, shown where green reaches the simplex boundary) to stay in the simplex

In the next experiment, we performed PCA on ILR and ALR-transformed probabilistic segmentation maps of 20 subjects from BrainWeb [21]. For each subject and at each pixel, 12 probability values capture the pixel's fuzzy membership in 12 classes such as cerebrospinal fluid (CSF), gray matter (GM), and white matter (WM). The mid-sagittal plane of all subjects are transformed to ILR and ALR space. After performing PCA in those spaces, new probability 


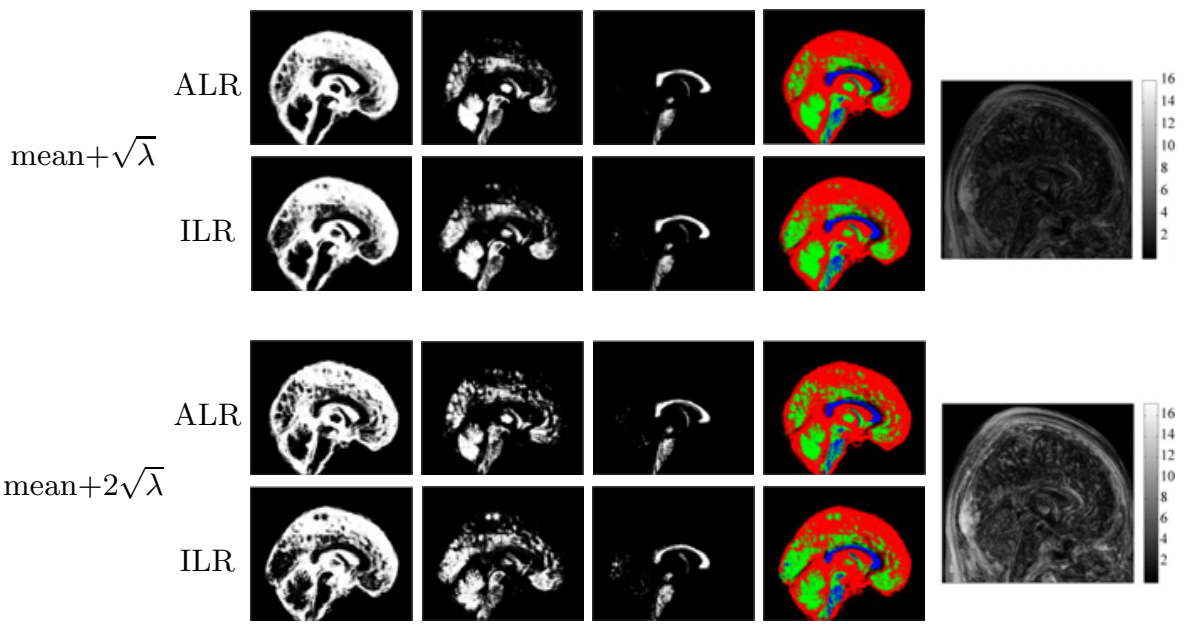

Fig. 5. Columns 1-3 show the variability in the resulting probability maps for CSF, GM, and WM. The 4th column uses the first three columns as RGB channels. $\lambda$ is the variance explained by the first mode. The 5th columns shows the simplicial distances (errors when using ALR (logOdds)) between the two probability maps resulting from ILR and ALR at each pixel.

maps along the first mode of variation are obtained and shown in Figure 5 , Different images obtained using ILR and ALR transformations show that the error from ALR can be avoided using the proposed method. The simplicial distances between pixels of the the new probability maps using ILR and ALR transformations are calculated and also shown in Figure 5.

\section{Conclusions}

We proposed an alternative probabilistic multi-shape representation: Isometric Log-Ratio. It has several desired properties: forms a vector space, isometric and thus isomprphic to the probability simplex, and results in a non-singular covariance. These properties do not exist together in any previously offered probabilistic computational anatomy work. We demonstrated how the lack of some of these properties degrades the results, e.g. statistical analysis using linear PCA. In the future, we intend to apply the method within the context of clinical applications.

\section{References}

1. Udupa, J.K., Grevera, G.J.: Go digital, go fuzzy. Pattern Recognit. Lett. 23(6), 743-754 (2002)

2. Zhang, Y., Brady, M., Smith, S.: Segmentation of brain MR images through a hidden Markov random field model and the expectation-maximization algorithm. IEEE Trans. Med. Imaging 20(1), 45-57 (2001) 
3. Grady, L.: Random walks for image segmentation. IEEE Trans. Pattern Anal. Mach. Intell. 28(11), 1768-1783 (2006)

4. Warfield, S., Zou, K., Wells, W.: Simultaneous truth and performance level estimation (STAPLE): an algorithm for the validation of image segmentation. IEEE Trans. Med. Imaging 23(7), 903-921 (2004)

5. Tsai, A., Wells, W., Tempany, C., Grimson, E., Willsky, A.: Mutual information in coupled multi-shape model for medical image segmentation. Med. Image Anal. 8(4), 429-445 (2004)

6. Babalola, K., Cootes, T.: Registering richly labelled 3D images. In: IEEE ISBI, pp. 868-871 (2006)

7. Leventon, M.E., Grimson, W.E.L., Faugeras, O.: Statistical shape influence in geodesic active contours. In: IEEE CVPR, pp. 316-323 (2000)

8. Golland, P., Grimson, W., Shenton, M., Kikinis, R.: Detection and analysis of statistical differences in anatomical shape. Med. Image Anal., 69-86 (2005)

9. Pohl, K.M., Fisher, J., Bouix, S., Shenton, M., McCarley, R.W., Grimson, W.E.L., Kikinis, R., Wells, W.M.: Using the logarithm of odds to define a vector space on probabilistic atlases. Med. Image Anal. 11(5), 465-477 (2007)

10. Malcolm, J., Rathi, Y., Shenton, M., Tannenbaum, A.: Label space: A coupled multi-shape representation. In: Metaxas, D., Axel, L., Fichtinger, G., Székely, G. (eds.) MICCAI 2008, Part II. LNCS, vol. 5242, pp. 416-424. Springer, Heidelberg (2008)

11. Hamarneh, G., Changizi, N.: Barycentric label space. In: MICCAI Workshop on Probabilistic Models for Medical Image Analysis, pp. 162-173 (2009)

12. Cremers, D., Schmidt, F.R., Barthel, F.: Shape priors in variational image segmentation: Convexity, Lipschitz continuity and globally optimal solutions. In: IEEE CVPR, pp. 1-6 (2008)

13. Aitchison, J.: The statistical analysis of compositional data. Chapman \& Hall Ltd., Boca Raton (1986)

14. Pawlowsky-Glahn, V., Egozcue, J.J., Tolosana-Delgado, R.: Lecture notes on compositional data analysis (2007), http://hdl.handle.net/10256/297

15. Egozcue, J.J., Pawlowsky-Glahn, V., Mateu-Figueras, G., Barcel-Vidal, C.: Isometric logratio transformations for compositional data analysis. Math. Geol. 35(3), 279-300 (2003)

16. Pawlowsky-Glahn, V., Egozcue, J.J.: Geometric approach to statistical analysis on the simplex. Stochastic Environmental Research and Risk Assessment 15(5), 384-398 (2001)

17. Pawlowsky-Glahn, V., Egozcue, J.J.: BLU estimators and compositional data. Math. Geol. 34(3), 259-274 (2002)

18. Billheimer, D., Guttorp, P., Fagan, W.F.: Statistical analysis and interpretation of discrete compositional data. Technical Report 11, University of Washington, Seattle, Washington (1997)

19. Billheimer, D., Guttorp, P., Fagan, W.F.: Statistical interpretation of species composition. J. of the American Statistical Association 96(456), 1205-1214 (2001)

20. Connor, R.J., Mosimann, J.E.: Concepts of independence for proportions with a generalization of the dirichlet distribution. J. of the American Statistical Association 64(325), 194-206 (1969)

21. Cocosco, C.A., Kollokian, V., Kwan, R.K.S., Pike, G.B., Evans, A.C.: BrainWeb: Online interface to a 3D MRI simulated brain database. NeuroImage 5, 425 (1997) 